\title{
Propuesta metodológica del indicador "Rango de Felicidad Local": asociación de indicadores relativos a la felicidad, bienestar y estado de ánimo
}

\author{
García Leyva, Alberto \\ Universidad Veracruzana, México \\ albergarcia@uv.mx
}

Resumen - Se aborda el tema de la felicidad y bienestar para proponer el indicador Rango de Felicidad Local, que agrupa variables del INEGI y el Banco de México, así como el World Happiness Report de la Red de Soluciones de Desarrollo Sostenible de las Naciones Unidas y otras fundaciones. La prueba piloto calculada para el estado de Veracruz, México, arroja un resultado de $46.23 \%$, el indicador tiene cualidades adaptativas, se puede calcular diariamente, y provee información a los tomadores de decisiones.

Palabras clave - Rango de Felicidad Local; Felicidad; Bienestar; Indicador;

\begin{abstract}
The topic of happiness and well-being is approached to propose the Rank of Local Happiness indicator, which groups variables from INEGI and Banco de México as well as the World Happiness Report of the United Nations Sustainable Development Solutions Network and other foundations. The pilot test calculated for the state of Veracruz, Mexico, yields a result of $46.23 \%$, the indicator has adaptive qualities, can be calculated daily, and provides information to decision makers.
\end{abstract}

Keywords - Rank of Local Happiness; Happiness; Well-being; Indicator;

\section{Interconectando Saberes, 2020}

ISSN: 2448-8704

\section{INTRODUCCIÓN}

Una de las características humanas inherentes al desarrollo de una vida digna es la felicidad, concepto pertinente y actual que tocan diferentes sectores de estudio que van desde la filosofía hasta la economía, pasando por la biología y las ciencias médicas.

Su definición se ha desarrollado a partir de diferentes aristas pertinentes, que, en su intento de describirla, han contribuido con la aportación de una serie de características conformantes, que ahora pueden ser utilizadas para un acercamiento a su medición.

Para fines de este artículo, la felicidad, bienestar y animosidad se entenderán como conceptos de igual significado, más allá de las diferencias conceptuales que puedan tener en general, son las características de cada concepto lo que enriquece

Fecha de Recepción: 03 de abril de 2020

Fecha de Aceptación: 07 de julio de 2020

Fecha de Publicación: 24 de julio de 2020 
los elementos a tener en consideración para el diseño y posterior medición del indicador aquí propuesto.

\section{¿QUÉ ES LA FELICIDAD?}

El tema de la felicidad resulta ser de El origen del presente artículo se remonta a la necesidad de mostrar de manera cuantificable el rango de felicidad que se puede tener de manera local, con la intensión de poder comparar los resultados con el contexto en el que se desarrolla, y así poder visualizar cuales son los factores que influyen en el sentimiento de las personas.

Es objetivo de este artículo, proponer un nuevo indicador que verse sobre la medición de la felicidad a escala local/regional de las personas que habitan dicho espacio geográfico, que tenga la capacidad de resumir los diferentes esfuerzos que se han realizado a partir de distintos organismos e instituciones, para tratar de medir el bienestar y felicidad de la población, por lo que se propone en este artículo, un mecanismo factible y realizable que cumpla con características de adaptación para cualquier ciudad del mundo, de contar con la información pertinente, y que se pueda calcular de manera inmediata con aplicaciones y/o monitoreos que pueden llegar a ser de frecuencias diarias.

La metodología del indicador propuesto agrupa información relativa proveniente de organismos institucionales tales como la Red de Soluciones de Desarrollo Sostenible de las Naciones Unidas, el Instituto Nacional de Estadística y Geografía (INEGI) y el Banco de México (BANXICO) y arroja un resultado numérico en forma de porcentaje. ilustraciones pertenecientes a la filosofía, ya que se trata de una deseo universal, y con el objetivo de hacer que el concepto de felicidad sea más tangible y desarrollable, han existido una serie de publicaciones que despliegan el tema, aunque la temática fue apartada de los estudios de las ciencias relativas a la sociedad y la psicología en un periodo de tiempo, en consecuencia se dio paso a diversos estudios científicos que abordaban el tema (López y Fierro, 2015).

La felicidad para Aristóteles es una acción que va con la razón, con la autorrealización de la persona, que se concibe feliz a través de actuar bien, de vivir lo mejor posible, versa que las buenas acciones tengan un impacto en el sujeto para que este bien, también para Aristóteles es propio al ser humano ser justo, por lo tanto, será feliz si consigue su función propia (Bosch, 2019).

La felicidad se puede entender también como un derecho, explica Alvarado (2016) que han existido una serie de generaciones en materia de derechos humanos empezando por aquellos acuñados en la revolución francesa, que versaban sobre la autonomía de las personas frente al estado, entendida como la primera generación, la segunda generación afianzada sobre garantizar los medios para desarrollar la autonomía necesaria a través de derechos humanos sustantivos a lo económico y 
social, por lo que la tercera generación refiere al progreso de una nueva fase en donde se encuentra el derecho a la felicidad, en la tercera generación de derechos humanos.

\section{Max Neef en su obra Desarrollo a Escala Humana} menciona que "no parece existir correlación entre los niveles alcanzados de crecimiento económico y la felicidad relativa de la gente involucrada" (Max Neef, 1993, p.72). Es de suma importancia mencionar que la felicidad parece no tener relación directa con fenómenos económicos.

Flores, Muñoz y Adrián (2018) mencionan que, de manera general, la felicidad 0 bienestar hace referencia a un desempeño y práctica óptima psicológica. Aunque existe debate sobre lo que se considera óptimo, por lo que se tiene que tomar a consideración las acciones de las personas para alcanzar la felicidad ya que estas están relacionadas a la definición que esta persona le dé, además el bienestar y la felicidad están enfocadas a dos sectores; el paradigma eudaimónico y el hedonista (Ryan y Deci 2001 en Flores, Muñoz y Adrián 2018). El segundo haciendo referencia a que la felicidad depende de desarrollar experiencias placenteras y el eudaimónico que hace relación por medio del potencial humano, no solamente mediante el placer (Flores, Muñoz y Adrián 2018). Para Flores, Muñoz y Adrián en su investigación, el concepto de felicidad y bienestar son considerados como sinónimos.
Los conceptos relativos al bienestar subjetivo, felicidad, afectividad positiva, calidad de vida se han utilizado de manera intercambiable, lo que ha generado una serie de diferentes escalas para evaluar teóricamente a la felicidad y sus dimensiones y tópicos (Vera, Celis y Córdova, 2011).

Se puede mencionar cuales son las condiciones necesarias para desarrollar la felicidad, de las que se encuentran; la libertad, amor, salud y comodidad económica, no obstante, puede existir infelicidad, ya que son condiciones generales necesarias pero insuficientes, la felicidad es una valoración personal y subjetiva que puede cambiar dependiendo el tipo de sociedad y la época (Margot, 2007).

Villoria (2011) en su investigación sobre el buen gobierno, menciona que Nozick, 1974 se hacia el cuestionamiento sobre si la felicidad era equivalente para todas las personas, tomando en cuenta las grandes diferencias que existen entre estas, ejemplo Marilyn Monroe y Einstein (Nozick, 1974 en Villoria, 2011).

La felicidad, aunque es algo que se busca ampliamente se desarrolla de manera diferente, hay distintos aspectos como las amistades, trabajo, salud, estudios y amor, en donde se tiene la creencia que los individuos felices tienen éxito por medio de éstos (Salavera y Usán 2017). 
Es interesante ver como diferentes investigaciones abordan el tópico de la felicidad y su intento por definirla. El concepto se desarrolla para diferentes tipos de aplicaciones y en el camino van forjando elementos clave para tener en cuenta en el momento de su medición.

A palabras de Caballero y Sánchez (2018) la felicidad es algo que se puede aprender y enseñar, que resulta tener beneficios para el desarrollo personal, profesional, social y académico, por lo que es imperioso acondicionar a los estudiantes para la vida profesional, se tenga una educación encaminada a la felicidad, mencionan Caballero y Sánchez en su investigación sobre la felicidad en estudiantes universitarios.

Borrero-Caldas, 2010; Carr, 2007 en Pozos, Rivera, Reyes y López (2013) señalan desde una perspectiva económica de la felicidad, que el dinero sirve para alcanzarla personalmente, pero solo lo correspondiente para satisfacer las necesidades primordiales tales como los servicios domésticos, sustento, vestido, y alimentación, pero una vez que estos son cubiertos, el contar con más dinero, ya no hace más feliz a la persona.

\section{Alarcón 2009 en Caycho y Castañeda (2015)} menciona que la felicidad es un estado pleno de satisfacción subjetiva cuando una persona logra obtener un bien deseado. Por lo que se concluyen en cuatro diferentes aspectos tomando en cuenta lo anterior; 1) sólo la persona desde su interior puede vivir la felicidad, 2) al tratarse de un estado, este es temporal, puede durar o no, 3) la felicidad llega cuando se posee el bien, 4) estos bienes pueden ser de diferente naturaleza, ya que pueden ser materiales, sociales, religiosos, estéticos, éticos y religiosos (Caycho y Castañeda, 2015).

Dicho lo anterior existen una serie de estudios muy diversos desde las diferentes disciplinas, que intentan abonar a la comprensión del tema y de paso definen la felicidad como base exploratoria primaria que da inicio a un análisis más profundo, haciendo uso de conceptos, técnicas y metodologías propias de cada disciplina.

Por ejemplo, un estudio que versa sobre la felicidad y rasgos de personalidad, desde el campo de la psicología de Morán, Fínez y Fernández (2017), desde el campo económico Jiménez, Martín y Montero (2014) en su investigación sobre la felicidad y la crisis económica en Andalucía, también la investigación de Veloso, Cuadra, Antezana, Avendaño y Fuentes (2013) sobre la relación entre inteligencia emocional, satisfacción vital, felicidad subjetiva y resiliencia en un estudio en donde hacen uso de instrumentos para medir las diferente variables.

Existen ciertos rangos en tomar en cuenta en los que se ubica las características de la felicidad, estos expuestos por diferentes aristas en el campo de la investigación, que deberán considerarse en el momento de la medición, así como también las herramientas y resultados que ya se encuentran disponibles. 
Continuando con algunos estudios que consideran el tema de la felicidad, se encuentra que, en la revisión bibliográfica realizada para esta investigación, los estudios se enfocan en un sector especial de la población y las metodologías utilizadas para abordar el tema, están relacionadas con encuestas, escalas o análisis descriptivos, en un periodo de tiempo determinado.

Sánchez de Gallardo y Pirela de Faría, (2017) realizan un estudio para comprobar el efecto de una intervención psicológica utilizando una escala de felicidad. Londoño (2011) efectúa una investigación por medio de una encuesta que mide la impresión del ingreso, la movilidad y justicia social en la felicidad, en donde primero realiza un análisis descriptivo sobre el impacto de características socioeconómicas sobre la movilidad.

El estudio de Jiménez, Zagalaz, Molero, Pulido y Ruiz (2013) en donde concluyen que los adolescentes que presentan buenas condiciones en materia aeróbica son también los más felices y presentan una elevada satisfacción de vida esto mediante un estudio de análisis estadístico. Sánchez y Méndez (2011) en su estudio sobre felicidad y bienestar subjetivo entre mujeres $y$ hombres, y la investigación de Núñez, González y Realpozo (2015) que versa sobre la relación entre felicidad y autoestima en estudiantes interculturales.

La importancia de estos estudios realizados abona al estudio de la felicidad y sus componentes para un sector en específico, que arroja resultados enriquecedores. Otra alternativa de tomar en cuenta es la relativa a los estudios de la felicidad en los grandes agregados sociales, plasmados en indicadores que tratan de medir mediante una serie de escalas la felicidad y bienestar en un espacio geográfico determinado, como países o regiones. A continuación, se expondrán una serie de indicadores internacionales y nacionales de los cuales se compondrá el indicador aquí propuesto "Rango de Felicidad Local", con el objetivo de resumir las diferentes propuestas en una escala de fácil comprensión y manejo para el público en general que además tenga facultades adaptativas y atemporales, que se pueda calcular para cualquier lapso, y con la capacidad de estimarse para diferentes regiones.

\section{World Happiness Report de la Red de Soluciones de Desarrollo Sostenible de las Naciones Unidas en asociación con distintas Fundaciones}

Uno de los indicadores metodológicos que han cobrado relevancia en los últimos años en materia de felicidad, ha sido el World Happiness Report (WHR) de la Red de Soluciones de Desarrollo Sostenible de las Naciones Unidas en colaboración con distintas fundaciones, este reporte se publica anualmente y enlista a los países conforme a su resultado.

El último reporte del año 2020, el octavo desde su inicio, tiene el objetivo que se ha perseguido en los demás reportes, que versa sobre comprender y medir el bienestar subjetivo para más de 150 países, para el último reporte, se enfocan a los entornos de la felicidad, tomando el periodo que va 
del 2017 al 2019, el reporte se conforma por 6 variables; el apoyo social, la ausencia de corrupción, la libertad, PIB per cápita, la generosidad y la esperanza de vida saludable, las calificaciones de estas variables no están basadas en índices relativos a la misma sino más bien en una encuesta de evaluación de sus vidas en donde los participantes responden según la escalera de Cantril y su posición en una escala del 0 al 10, 0 siendo la peor vida posible, también se agrega un séptimo segmento llamado distopía que contiene valores iguales a los peores promedios del mundo para las 6 variables, como si fuera un país, esto se utiliza como punto de referencia para que los países reales contribuyan de manera positiva en las variables (Helliwell, John F., et al., 2020).

El resultado para el caso de México fue de 6.46 ubicándolo en la posición número 24 , en la gráfica 1 que se muestra a continuación, se puede observar los 12 primeros lugares con sus puntuaciones, así como algunos países seleccionados correspondientes al continente americano e Hispanoamérica, para mostrar un total de los primeros 25 países seleccionados. Se expone el lugar en que quedó el país, seguido del nombre y la gráfica con su calificación.
Gráfica 1. Ranking de Felicidad (WHR) 2017 - 2019. Países Seleccionados.

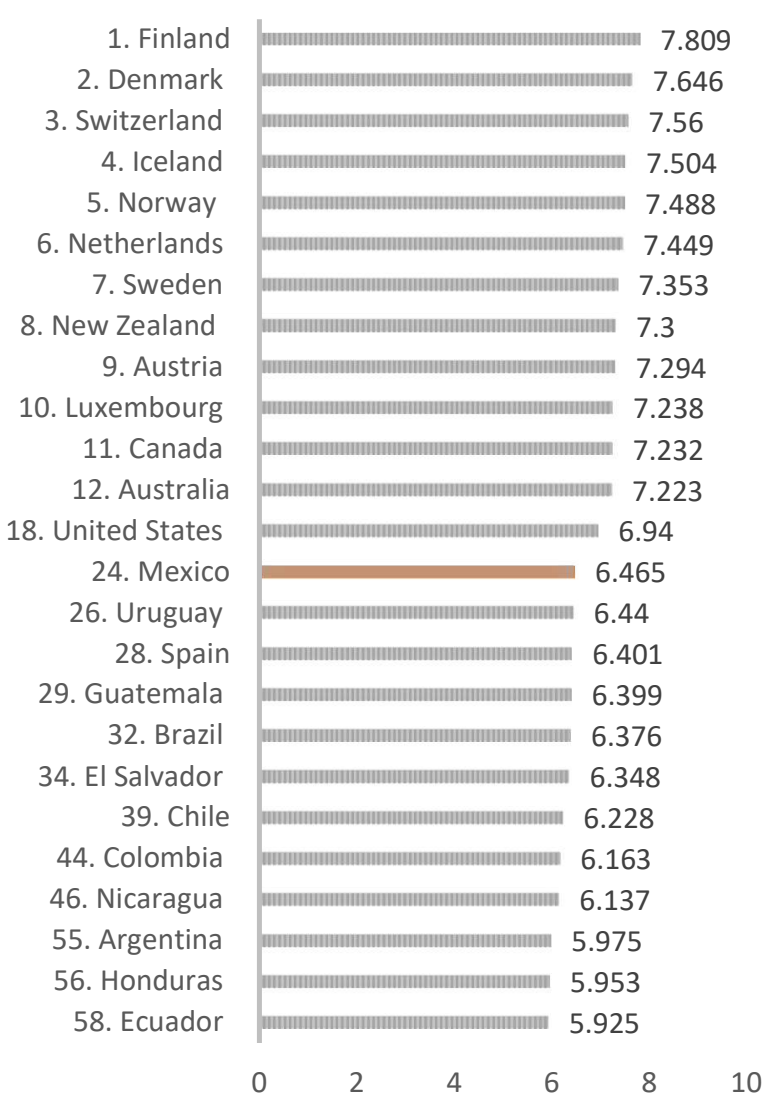

Fuente: Elaboración propia con información de: Helliwell, John F., Richard Layard, Jeffrey Sachs, and Jan-Emmanuel De Neve, eds. 2020. World Happiness Report 2020. New York: Sustainable Development Solutions Network.

Como se puede observar en la gráfica anterior, el país que ocupa la primera posición es Finlandia con un resultado de 7.80 , seguido de Dinamarca y Suiza con 7.64 y 7.56 respectivamente, como ya se mencionó México en el lugar 24 con 6.46. Sin duda una herramienta muy valiosa al momento de conformar un indicador de felicidad a escala regional/local. 


\section{Bienestar subjetivo BIARE del INEGI}

El Instituto Nacional de Estadística y Geografía (INEGI) cuenta con una herramienta que se puede consultar en línea llamado Bienestar Subjetivo BIARE, el cual presenta una serie de información a manera de estadísticas que tratan sobre el bienestar de la población en México. La información presentada en este artículo de investigación proviene de lo publicado en la página de internet INEGI, S.F., detalles en la bibliografía. Aunque también se sugiere revisar INEGI, 2015.

La iniciativa de medición del progreso social, surge como consenso internacional a partir de reconocer las limitaciones que tienen las estadísticas convencionales para medirlo, y comenzando por una serie de recomendaciones por parte del organismo internacional de la Organización para la Cooperación y el Desarrollo Económico (OCDE), se formuló el denominado Bienestar Subjetivo, que bajo la orientación de Medición del Progreso de las Sociedades, se pueden encontrar dos enfoques complementarios, el bienestar objetivo, basado en satisfacción material, y el subjetivo enfocado a vivencias de las personas, ante esta circunstancia surge los módulos de Bienestar Autorreportado (BIARE) de encuestas en los hogares, para visualizar como impactan las políticas públicas en sus vidas, con el objetivo de generar información de manera periódica y que pueda ser comparada con países de la OCDE, el mecanismo de aplicación de la encuesta es mediante un formulario compuesto por una serie de reactivos (INEGI, S.F.)
La información pertinente se publica en la página web del INEGI, en la parte de investigaciónestadísticas experimentales- bienestar subjetivobásico, los meses de Enero, Abril, Julio y Octubre y dentro de la información se puede encontrar los siguientes contenidos en forma de cuadros de datos prácticos; Población según nivel de satisfacción: general y por dominios específicos, y Población según nivel de balance afectivo: general y específicos, mismos que se encuentran también disponibles en porcentajes.

De acuerdo con el Cuadro 1 mostrado en el BIARE (INEGI, 2020a) de Población según nivel de satisfacción: general y por dominios específicos de Enero del 2020, exponen una serie de dominios de satisfacción compuestos por; vida en general, nivel de vida, estado de salud, logros en la vida, relaciones personales, perspectivas a futuro, tiempo libre, seguridad ciudadana, actividad $u$ ocupación, vivienda, ciudad y país, calificados en cuatro diferentes rangos; insatisfecho (que va de 0 a 4), poco satisfecho (que va de 5 a 6), moderadamente satisfecho (de 7 a 8 ) y satisfecho (9 a 10) posteriormente se encuentra el mismo cuadro pero expresado en porcentaje (INEGI, 2020a).

\section{Estado de ánimo de los tuiteros en México del INEGI}

El Estado de ánimo de los tuiteros en México del INEGI, un indicador cuyos números se publican desde el primero de enero del 2016, y muestra una serie de gráficas con resultados que se van actualizando diariamente. 
Bajo la premisa que la información del internet contribuye a generar información estadística, el INEGI incursiona a problemáticas de Big Data con el que determina el estado de ánimo de los tuiteros en México, el Instituto recopila tuits georreferenciados y públicos mediante componentes de Twitter y clasifica cada tuit por medio del contenido emotivo del usuario cuando publico el tuit, posteriormente pasa por un proceso de normalización y limpieza de los tuits para ser divididos mediante algoritmos novedosos para clasificar y procesar la información, se establece el lugar de residencia para diferenciar el usuario local con el visitante, y se estima un índice que significa la relación de los tuits positivos entre los tuits negativos que es publicado a nivel nacional $y$ estatal, también se publica la información de manera diaria, y se muestra la información a través de mapas y graficas (INEGI, 2017).

En la página web del indicador, INEGI (2020b), se muestra en una primera gráfica, los resultados generales del estado de ánimo en México, misma donde se puede observar una gráfica de líneas con resultados mixtos pero con pendiente negativa y con tendencia igualmente negativa, en dicha gráfica se muestra el valor del índice y la relación entre tuits negativos y positivos, tanto en números brutos como en porcentaje, esto en un cuadro resumen que aparece al poner el cursor sobre el punto de la gráfica, la información se presenta desde enero del 2016 hasta la fecha de consulta actual, para el caso de esta investigación el 26 de marzo del 2020, posteriormente se puede seleccionar más información que también es presentada a escala estatal a través de un mapa e información adicional como la de recolección de datos, publicada por medio de una gráfica de líneas.

\section{EL RANGO DE FELICIDAD LOCAL}

Como se pudo observar con anterioridad, existen una serie de herramientas disponibles que tratan de medir el bienestar subjetivo, la felicidad y el estado de ánimo, la información recopilada por las instituciones presentan una exploración metodológica muy profunda y los resultados se perciben confiables, para la conformación del Rango de Felicidad Local es indispensable que sus componentes tengan estas características, por lo que este nuevo indicador estará conformado por estas variables.

En contexto y tomando en cuenta los conceptos teóricos expresados con anterioridad en esta investigación, se tomaron variables que miden el bienestar, felicidad y estado de ánimo que son expuestos de forma entendible para el público en general, de la misma manera las herramientas que se utilizan para procesar y calcular la información secundaria son de alcance masivo, lográndose calcular en programas básicos de cálculo, ya que el objetivo final consta que la metodología se pueda replicar para otras localidades con relativa facilidad.

Se tienen en una primera instancia 3 variables que miden las características necesarias y presentan diferentes escalas geográficas y son 
publicadas/actualizadas en diferentes periodos de tiempo, como se muestra en el Cuadro 1, los espacios de temporalidad de cada estudio corresponden a la publicación propia que cada organismo tiene.

\section{Cuadro 1. Escala geográfica y temporalidad de} publicación de las variables seleccionadas.

\begin{tabular}{|c|c|c|}
\hline Variable & $\begin{array}{c}\text { Escala geográfica } \\
\text { nivel }\end{array}$ & $\begin{array}{l}\text { Temporalidad de } \\
\text { actualización }\end{array}$ \\
\hline $\begin{array}{c}\text { World } \\
\text { Happiness } \\
\text { Report } \\
\end{array}$ & Nacional & Anual \\
\hline $\begin{array}{c}\text { Bienestar } \\
\text { subjetivo } \\
\text { BIARE Básico }\end{array}$ & Nacional & Trimestral \\
\hline $\begin{array}{l}\text { Estado de } \\
\text { ánimo de los } \\
\text { tuiteros en } \\
\text { México }\end{array}$ & Nacional/Estatal & Diario \\
\hline
\end{tabular}

Es necesario incluir una variable adicional que muestre el panorama y la percepción del entorno económico de la región, más allá del componente del World Happiness Report con el PIB per cápita, por lo que se tomará como variable la respuesta a la pregunta; Actualmente la economía está mejor que hace un año de la percepción del entorno económico del documento "Encuesta sobre las Expectativas de los Especialistas en Economía del Sector Privado" del Banco de México, que se publica para cada mes y que se puede consultar a través de la página web en Banco de México (2020a). Dicha variable se expresa en porcentaje de las respuestas de "si y no" y es la perspectiva de la economía del país con respecto al año anterior, se entiende que es a escala general. Para el caso de la conformación de este indicador se tomará en cuanta el porcentaje de la respuesta positiva.
Para la integración del Rango de Felicidad Local se tomarán así cuatro variables, en sus escalas nacionales, salvo el de Estado de ánimo de los tuiteros en México que se tomara a nivel estatal, con su temporalidad de actualización vista en el Cuadro 1. Por lo que el indicador se puede actualizar de manera diaria y se puede adaptar para los 32 estados de la República Mexicana en una primera instancia, para el caso de ejemplo y prueba piloto mostrado en este artículo, se tomará el estado de Veracruz y se calculará un promedio de los datos arrojados en estas cuatro variables; esto es,1) la calificación de México en la WHR, expresada en porcentaje 2) respuesta a vida en general, nivel satisfecho (9-10), en porcentaje de "Población según nivel de satisfacción: general y por dominios específicos" del Bienestar Subjetivo en México. Módulo BIARE Básico del INEGI, 3) los tuits positivos en porcentaje para el estado de Veracruz (prueba piloto) del día de la consulta y 4) el resultado positivo de "Actualmente la economía está mejor que hace un año" de la percepción del entorno económico del documento del Banco de México antes explicado.

Por lo que la conformación del Rango de Felicidad Local se determina de la siguiente manera;

$$
\text { Rango de Felicidad Local }=\frac{\sum(W H R+B I A R E+T W+E B M)}{\mathrm{N}}
$$

WHR= \% World Happiness Report

BIARE= Vida en general, nivel satisfecho (9-10), en \% de "Población según nivel de satisfacción: general y por dominios específicos" Bienestar Subjetivo en México, INEGI. 
TW= tuits positivos en porcentaje, INEGI.

$\mathbf{E B M}=$ el resultado positivo de "Actualmente la economía está mejor que hace un año", Banco de México.

$\mathbf{N}=$ Número de variables seleccionadas. 4

Para el caso de la prueba piloto calculada para el estado de Veracruz, la estimación quedaría de la siguiente manera;

\section{Cuadro 2. Resultados de las variables a 28 de} marzo del 2020

\begin{tabular}{|c|c|c|c|c|}
\hline Variable & Resultado & $\begin{array}{c}\text { Útimo } \\
\text { periodo } \\
\text { consultado }\end{array}$ & Fuente & $\begin{array}{c}\text { Nivel } \\
\text { geográfico }\end{array}$ \\
\hline WHR & $64.65 \%$ & $\begin{array}{l}\text { Reporte del } \\
2020\end{array}$ & $\begin{array}{l}\text { World } \\
\text { Happiness } \\
\text { Report } \\
\text { (Helliwell, } \\
\text { J., et al, } \\
\text { 2020). }\end{array}$ & Nacional \\
\hline BIARE & $48.28 \%$ & Enero 2020 & $\begin{array}{l}\text { Instituto } \\
\text { Nacional } \\
\text { de } \\
\text { Estadística } \\
\text { y } \\
\text { Geografía } \\
(2020 a) .\end{array}$ & Nacional \\
\hline TW & $60 \%$ & $\begin{array}{l}27 \text { de } \\
\text { marzo } 2020\end{array}$ & $\begin{array}{l}\text { Instituto } \\
\text { Nacional } \\
\text { de } \\
\text { Estadística } \\
\text { y } \\
\text { Geografía } \\
\text { (2020b). }\end{array}$ & Estatal \\
\hline EBM & $12 \%$ & $\begin{array}{l}\text { Encuesta } \\
\text { de febrero } \\
\text { del } 2020\end{array}$ & $\begin{array}{l}\text { Banco de } \\
\text { México } \\
\text { (2020b). }\end{array}$ & Nacional \\
\hline
\end{tabular}

Fuente: Elaboración propia a partir de fuentes primarias antes mencionadas en el documento.

Una vez homogenizados los resultados y estimados a porcentajes, para la variable que no lo estaba, se procede a calcular el Rango de Felicidad local piloto, cabe resaltar que para este ejercicio de prueba se toman los últimos reportes, encuestas y resultados disponibles, más allá de tomar los resultados para un periodo de tiempo específico en concordancia, la idea central es calcular el indicador con la información más reciente disponible. Para esta ocasión, el resultado debe ser interpretado como correspondiente al periodo enero-marzo.

Rango de Felicidad Local $=\frac{\sum(64.65+48.28+60+12)}{4}$

Rango de Felicidad Local $=\mathbf{4 6 . 2 3} \%$

En términos de interpretación se tiene un resultado final de Rango de Felicidad Local de $46.23 \%$, es decir, para el estado de Veracruz en una escala del 1 al 100 , las personas tienen un rango de felicidad radicado en su estado de ánimo, su bienestar subjetivo, entorno económico y felicidad en general para el periodo enero-marzo en resultado adverso por debajo de la medida mayoritaria del $50 \%$.

\section{CONCLUSIÓN}

El Rango de Felicidad Local es un indicador que agrupa como variables, información de suma importancia proveniente de instituciones reconocidas como el INEGI y el Banco de México, así como también el resultado del prestigioso WHR de la Red de Soluciones de Desarrollo Sostenible de las Naciones Unidas en colaboración con distintas fundaciones. El resultado es un promedio evaluativo de la felicidad en general, tomando como esta, componentes como el bienestar, el estado de ánimo y la percepción del entorno.

En este artículo de investigación se realizó una prueba piloto para el estado de Veracruz, pero el 
indicador tiene cualidades adaptativas, para ser calculado para cualquier estado de la República Mexicana o región del mundo, de tener información disponible que concuerde con la de las fuentes aquí tomadas, y que además este indicador puede ser calculado y monitoreado de manera diaria.

Es indispensable calcular el Rango de Felicidad Local para futuras investigaciones a lo largo de un lapso, para dar cuenta así, si existe alguna correlación con otros indicadores o contextos en lo que se ve envuelta una sociedad, identificar los factores que influyen en el comportamiento de esta.

El Rango de Felicidad Local sirve, además, para suministrar información a los tomadores de decisiones, sobre cuáles son los factores que pueden influir en el indicador. El objetivo de las políticas públicas, trabajadas desde el Gobierno Federal de México, se enfocan sobre mejorar el bienestar de las personas. El indicador aquí propuesto abona para ser un mecanismo de medición del cumplimiento de tal objetivo. Se puede tratar de una herramienta que se pone a disposición de tomadores de decisiones como método evaluativo de políticas públicas. Versa también, sobre un intento de colaborar desde la academia, para que el propósito final de la felicidad en las personas se pueda alcanzar.

\section{REFERENCIAS}

Alvarado, José Tomás (2016). ¿Derecho a la felicidad? Díkaion, 25(2), 243-265. [fecha de Consulta 20 de Marzo de 2020]. ISSN: 01208942. Disponible en:

http://www.redalyc.org/articulo.oa?id $=72048$ $\underline{894005}$

Banco de México (2020a). Encuestas sobre las expectativas de los especialistas en economía del sector privado. Reporte de resultados. Consultado el 28 de Marzo del 2020 en la página web; https://www.banxico.org.mx/publicaciones-yprensa/encuestas-sobre-las-expectativasde-los-especialis/encuestas-expectativasdel-se.html

Banco de México (2020b). Encuestas sobre las expectativas de los especialistas en economía del sector privado: febrero de 2020. Consultado el 28 de Marzo del 2020 en la página web; https://www.banxico.org.mx/publicaciones-yprensa/encuestas-sobre-las-expectativasde-los-especialis/\%7BA3A1A5FD-227FE474-C65B-AE19E9155414\%7D.pdf

Bosch Rabell, Magdalena (2019). La felicidad en Aristóteles: Fin, contemplación y deseo. SCIO. Revista de Filosofía, (16), 41-60. [fecha de Consulta 13 de Mayo de 2020]. ISSN: 1887-9853. Disponible en: https://dialnet.unirioja.es/servlet/articulo?codi go $=7054426$

Caballero-García, Presentación A., \& Sánchez Ruiz, Sara (2018). La felicidad en estudiantes universitarios. ¿Existen diferencias según género, edad o elección de estudios? Revista Electrónica Interuniversitaria de Formación del Profesorado, 21(3),1-18. [fecha de Consulta 23 de Marzo de 2020]. Disponible en: http://www.redalyc.org/articulo.oa?id=21705 $\underline{9711001}$ 
Caycho Rodríguez, Tomás, \& Castañeda, María Claudia (2015). Felicidad y optimismo en adolescentes y jóvenes peruanos y paraguayos: un estudio predictivo. Salud \& Sociedad, 3(6), 250-263. [fecha de Consulta 23 de Marzo de 2020]. Disponible en: https://www.redalyc.org/articulo.oa?id=43974 $\underline{4554005}$

Flores-Kanter, Pablo Ezequiel, \& Muñoz-Navarro, Roger, \& Adrián Medrano, Leonardo (2018). Concepciones de la Felicidad y su relación con el Bienestar Subjetivo: un estudio mediante Redes Semánticas Naturales. Liberabit. Revista Peruana de Psicología, 24(1),115-130. [fecha de Consulta 20 de Marzo de 2020]. ISSN: 1729-4827.

Disponible en:

https://www.redalyc.org/articulo.oa?id=68656 $\underline{777008}$

Helliwell, John F., Richard Layard, Jeffrey Sachs, and Jan-Emmanuel De Neve, eds. (2020). World Happiness Report 2020. New York: Sustainable Development Solutions Network. ISBN: 978-1-7348080-0-1. Disponible en: https://worldhappiness.report/ed/2020/\#read

Instituto Nacional de Estadística y Geografía. INEGI, (S.F.). Investigación- Estadísticas experimentales. Bienestar subjetivo BIARE. Consultado el 26 de Marzo del 2020 en la página web; https://www.inegi.org.mx/investigacion/biene star/basico/

Instituto Nacional de Estadística y Geografía. INEGI, (2020a). Bienestar Subjetivo en México. Módulo BIARE Básico. Enero, 2020. Recuperado el 26 de Marzo del 2020 de la página web;

https://www.inegi.org.mx/investigacion/biene star/basico/default.htm|\#Tabulados

Instituto Nacional de Estadística y Geografía. INEGI, (2020b). Estado de ánimo de los tuiteros en México. Consultado el 27 de Marzo del 2020 en la página web; https://www.inegi.org.mx/app/animotuitero/\#/ app/multiline
Instituto Nacional de Estadística y Geografía. INEGI, (2017). Estado de ánimo de los tuiteros en los Estados Unidos Mexicanos: documento metodológico / Instituto Nacional de Estadística y Geografía. -- 2da. Edición. -México: INEGI, c2018. Disponible en: http://internet.contenidos.inegi.org.mx/conten idos/Productos/prod serv/contenidos/espan ol/bvinegi/productos/nueva estruc/70282509 9718.pdf

Instituto Nacional de Estadística y Geografía. INEGI, (2015). Módulo de Bienestar Autorreportado 2015: BIARE: Documento Metodológico. Instituto Nacional de Estadística y Geografía. INEGI. México: INEGI, c2015. ISBN: 978-607-739-683-3.

Jiménez Aguilera, Juan de Dios, Martín Martín, José María \& Montero Granados, Roberto (2014). Felicidad, desempleo y crisis económica en Andalucía. Algunas evidencias. Revista de Estudios Regionales, (99), 183-207. [fecha de Consulta 23 de Marzo de 2020]. ISSN: 0213-7585.

Disponible en:

http://www.redalyc.org/articulo.oa?id=75531 $\underline{857007}$

Jiménez-Moral, Juan A.; Zagalaz Sánchez, María L.; Molero, David; Pulido-Martos, Manuel; Ruiz, Jonatan R (2013). Capacidad aeróbica, felicidad y satisfacción con la vida en adolescentes españoles. Revista de Psicología del Deporte, 22(2),429-436. [fecha de Consulta 24 de Marzo de 2020]. ISSN: 1132-239X. Disponible en:

https://www.redalyc.org/articulo.oa?id=23512 $\underline{8058011}$

Londoño Vélez, Juliana (2011). Movilidad social, preferencias redistributivas y felicidad en Colombia. Desarrollo y Sociedad, (68), 171212. [fecha de Consulta 24 de Marzo de 2020]. ISSN: 0120-3584. Disponible en: https://www.redalyc.org/articulo.oa?id=16912 $\underline{2461006}$ 
López, Juan Pablo, \& Fierro, Isidro (2015).

Determinantes de la felicidad en los

administradores: una investigación realizada

en las farmacias del Grupo Difare en

Ecuador. Universidad \& Empresa, 17(29),

181-211. [fecha de Consulta 20 de Marzo de

2020]. ISSN: 0124-4639. Disponible en:

https://www.redalyc.org/articulo.oa?id=18724

$\underline{3745008}$

Manfred Max-Neef. (1993). Desarrollo a Escala Humana. Conceptos, aplicaciones y algunas reflexiones. Montevideo, Uruguay: Editorial Nordan-Comunidad. ISBN: 84-7426-217-8.

Disponible en: https://www.maxneef.cl/libros/desarrollo a escala humana/

Margot, Jean-Paul (2007). LA FELICIDAD. Praxis Filosófica, (25), 55-79. [fecha de Consulta 23 de Marzo de 2020]. ISSN: 0120-4688.

Disponible en:

https://www.redalyc.org/articulo.oa?id=20901 $\underline{4642004}$

Morán, María Consuelo, \& Fínez, María José, \& Fernández-Abascal, Enrique G. (2017). Sobre la felicidad y su relación con tipos y rasgos de personalidad. Clínica y Salud, 28(2), 59-63. [fecha de Consulta 23 de Marzo de 2020]. ISSN: 1130-5274.

Disponible en:

https://www.redalyc.org/articulo.oa?id=18065 $\underline{1222003}$

Núñez Ramírez, Marco Alberto; González Quirarte, Gloria Esthela; Realpozo Reyes, Rosario del Carmen (2015). Relación entre autoestima y felicidad desde la psicología positiva en estudiantes de enfermería intercultural. Enfermería Actual en Costa Rica, (29), [fecha de Consulta 24 de Marzo de 2020]. Disponible en: https://www.redalyc.org/articulo.oa?id=44839 779004
Pozos Gutiérrez, José Luis, Rivera Aragón, Sofía, Reyes Lagunes, Isabel, \& López Parra, María Sughey (2013). Escala de Felicidad en la Pareja: Desarrollo y Validación. Acta de Investigación Psicológica - Psychological Research Records, 3(3),1280-1298. [fecha de Consulta 23 de Marzo de 2020]. ISSN: 2007-4832. Disponible en:

https://www.redalyc.org/articulo.oa?id=35893 $\underline{3345008}$

Salavera Bordás, Carlos, \& Usán Supervía, Pablo (2017). Repercusión de las estrategias de afrontamiento de estrés en la felicidad de los alumnos de Secundaria. Revista Electrónica Interuniversitaria de Formación del Profesorado, 20(3),65-77. [fecha de Consulta 23 de Marzo de 2020]. Disponible en:

https://www.redalyc.org/articulo.oa?id=21705 $\underline{2050005}$

Sánchez Aragón, Rozzana, \& Méndez Canales, Roque (2011). Elementos mediadores de la felicidad y el bienestar subjetivo en hombres y mujeres. Revista Costarricense de Psicología, 30(45-46),51-76. [fecha de Consulta 24 de Marzo de 2020]. ISSN: 02571439. Disponible en:

https://www.redalyc.org/articulo.oa?id=47674 8709003

Sánchez de Gallardo, Marhilde, \& Pirela de Faría, Ligia (2017). Efecto de una intervención psicológica en la felicidad de estudiantes universitarios. Un estudio experimental. Omnia, 23(3), 76-94. [fecha de Consulta 24 de Marzo de 2020]. ISSN: 1315-8856. Disponible en:

https://www.redalyc.org/articulo.oa?id=73754 $\underline{947006}$

Veloso-Besio, Constanza; Cuadra-Peralta, Alejandro; Antezana-Saguez, Ivonne; Avendaño-Robledo, Rosa; Fuentes-Soto, Leslie (2013). Relación entre Inteligencia Emocional, Satisfacción Vital, Felicidad Subjetiva y Resiliencia en funcionarios de Educación Especial. Estudios Pedagógicos, XXXIX (2),355-366. [fecha de Consulta 23 de Marzo de 2020]. ISSN: 0716-050X.

Disponible en:

https://www.redalyc.org/articulo.oa?id=17352 $\underline{9673021}$ 
Vera-Villarroel, Pablo, \& Celis-Atenas, Karem, \& Córdova-Rubio, Natalia (2011). Evaluación de la Felicidad: Análisis Psicométrico de la Escala de Felicidad Subjetiva en Población Chilena. Terapia Psicológica, 29(1),127-133. [fecha de Consulta 20 de Marzo de 2020]. ISSN: 0716-6184. Disponible en:

http://www.redalyc.org/articulo.oa? $\mathrm{id}=78518$ $\underline{428013}$

Villoria, Manuel (2011). ¿Más libertad o más felicidad? El buen gobierno del siglo XXI. Revista del CLAD Reforma y Democracia, (51), 5-44. [fecha de Consulta 23 de Marzo de 2020]. ISSN: 1315-2378. Disponible en: https://www.redalyc.org/articulo.oa?id=35753 $\underline{3683001}$ 